connexion with vaccines. They ought very easily to resolve the question as to what is the best excipient for a vaccine-whether a menstruum which would restrain emigration, or one, like physiological salt solution, which would call forth a vigorous emigrating response at the point of injection. And lastly-and this would be one of the most important applications of observations on emigration-we ought to be able to determine what is in the case of each bacterial vaccine the dose which will induce the earliest possible and the most effective determination of phagocytes to the focus or foci of infection.

(To be continued.)

\section{ON THE PENETRATING POWER OF THE $X$ RAYS FROM THE COOLIDGE TUBE.}

\section{BY SIDNEY RUSS, D.Sc.}

A Coolidge $\mathrm{X}$ ray tube having been acquired by the electro-therapeutical department of the Middlesex Hospital, Mr. C. R. C. Lyster requested me to make some measurements of the radiation emitted from it, preparatory to its being put into clinical use. The results of a first systematic series of tests upon it were communicated to the February meeting of the Roentgen Society. The leading features of the observations were: (1) The recognition that the unscreened radiation from the bulb was heterogeneous, consisting of "soft," "medium," and "hard" rays; and (2) the relatively larger production of "hard" rays than of "soft" rays when the heating current, regulating the cathode stream, was increased.

Since the meeting referred to, the observations on the bulb have been continued with two objects in view-first, to measure the hardness of the rays which would be used clinically (for superficial and for deep-seated conditions) ; and, secondly, to find how nearly the most penetrating rays given out from the bulb approach the penetrating power of the hard gamma rays from radium. It was assumed that for superficial conditions unscreened "soft" and "medium" rays would be used, and that for deep-seated conditions the rays would be heavily filtered by aluminium; accordingly measurements were made of the character of the rays under these circumstances. This was done by finding how the intensity of the beam of $X$ rays was reduced on interposing sheets of aluminium between the bulb and a small gold-leaf electroscope used to measure this intensity.

When measuring the unscreened rays the bulb was run under a moderate sparking distance $(3 \mathrm{~cm}$. between spheres $5 \mathrm{~cm}$. in diameter, corresponding to about $11 \mathrm{~cm}$. between points). The gradual reduction in the intensity of the radiation as increasing thicknesses of aluminium are interposed is shown in Curve 1, Fig. 1 . When this curve is analysed it is found to consist of approximately three portions, which may be considered as Section D, in which the rays are "soft" and therefore easily absorbed; Section E, where they are rather more penetrating; and Section $\mathrm{F}$, where they are considerably "harder." The beam is, as stated, heterogeneous.

The observations upon heavily screened rays were made as follows. For the same heating current as before the spark-gap was increased to nearly the limit of the coil (viz., $10 \mathrm{~cm}$. between the spheres or $30 \mathrm{~cm}$. between points) and the rays were screened by $7 \mathrm{~mm}$. of aluminium. The character of the rays which penetrated this filter was found in the manner described, and the absorption suffered in going through various thicknesses of aluminium is shown in Curve 2 of the same figure. Analysis of this curve shows that the rays are very hard and practically of a homogeneous nature.

The introduction of as much as $7 \mathrm{~mm}$. of aluminium for the purpose of screening effects a considerable reduction in the intensity of the rays, but, granted that at least 3 or $4 \mathrm{~mm}$. are necessary it remains to determine whether the extra amount of aluminium, by which homogeneity is attained, entails too large a reduction in the quantity of rays available for use; the beam is not quite homogeneous with $4 \mathrm{~mm}$. screening. Actually it is found that this extra $3 \mathrm{~mm}$. entails a diminution of nearly 25 per cent. in the intensity of the beam already screened by $4 \mathrm{~mm}$. of aluminium. Apart from any desirable physiological effects which the hardest. $\mathrm{X}$ rays may give rise to, the use of a homogeneous. beam ensures the most uniform irradiation throughout the tissues that is possible, assuming that such. application has to be made externally; moreover, it allows of an accurate determination of the strength of the radiation at any particular depth of tissue.

The results represented in Fig. 1 were obtained by using aluminium as the absorbing substance for the rays; this is a matter of convenience, for the pure material is obtainable in sheets of any required thickness. When it is desired to apply the data to determine the gradual loss of intensity of $X$ rays as they make their way through the tissues certain precautions are necessary. If we consider that the

FIG. 1.

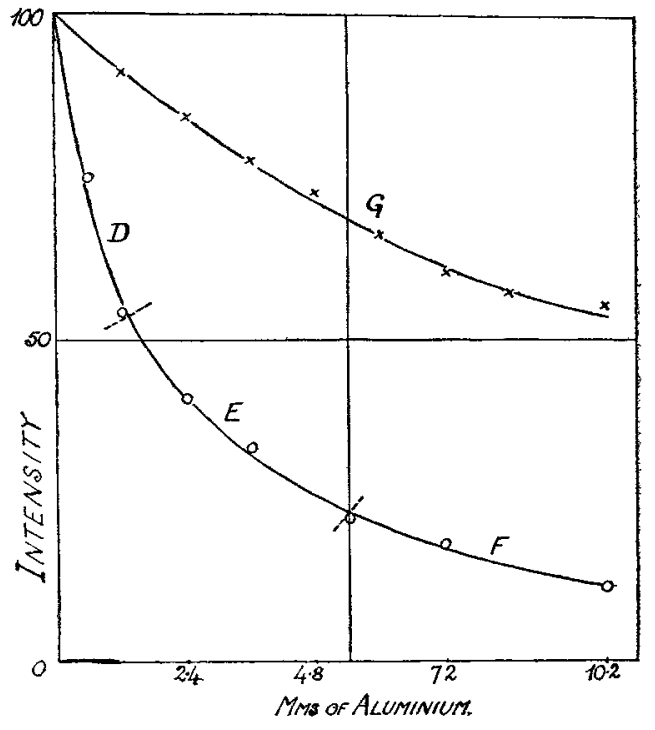

tissues of the human body have on an average about the same density as water, then if we knew the relative transparency of water and aluminium for $X$ rays of any specified quality we could gauge the absorption which the tissues would exercise upon any particular beam of $X$ rays.

When experiments are made to determine this relation between water and aluminium it is found that in the case of soft $X$ rays water (and therefore tissues also) absorbs them much less than its density would suggest, actually about one-fourth part; hence, since aluminium is $2 \cdot 7$. times the density of water, $1 \mathrm{~mm}$. of aluminium absorbs as much $4 \times 2.7=10.8 \mathrm{~mm}$. of tissue. As one passes to rays of a more penetrating character water becomes relatively to aluminium a better absorber, 
and when the homogeneous rays transmitted through $7 \mathrm{~mm}$. aluminium are reached, the factor $1 / 4$ has reached $3 / 4$, so that $1 \mathrm{~mm}$. of aluminium is now equivalent to $4 / 3 \times 2.7=3.6 \mathrm{~mm}$. of tissue.

The data are now available for calculating the degree to which the dose of $X$ rays administered varies from point to point in the tissues. As an illustration of this we may consider the case of some condition being treated by $\mathrm{X}$ rays screened in the manner indicated. Let it be assumed that the thickness of tissue is $16 \mathrm{~cm}$. and that the surface of the skin is $20 \mathrm{~cm}$. from the anodethe data in the accompanying table show what

Depth below surface.

0
$2 \mathrm{~cm}$.
$4, "$
$6, "$
$8, "$
$10 "$,
$12, "$
$14, "$
$16,$,

$$
\begin{array}{r}
\text { In } \\
100 \cdot \\
57 \cdot 6 \\
33 \cdot 8 \\
20 \\
12 \cdot 1 \\
7.2 \\
4.8 \\
1.8
\end{array}
$$$$
\text { Intensity of rays. }
$$$$
(a) \quad(b)
$$$$
\begin{array}{lll}
100 \cdot 0 & \ldots & 1.8
\end{array}
$$$$
\begin{array}{llll}
57 \cdot 6 & \ldots & 2 \cdot 8 \\
33 \cdot 8 & \cdots & 4 \cdot 5
\end{array}
$$$$
33.8 \quad \ldots .4 .5
$$$$
20 \cdot 0 \quad \cdots \quad 7 \cdot 2
$$$$
\begin{array}{lll}
12 \cdot 1 & \ldots & 12 \cdot 1
\end{array}
$$$$
\begin{array}{lll}
7 \cdot 2 & \ldots & 20 \cdot 0
\end{array}
$$

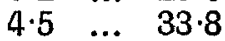$$
2 \cdot 8 \quad \ldots \quad 57 \cdot 6
$$$$
\begin{array}{llll}
1 \cdot 8 & \ldots & 100 \cdot 0
\end{array}
$$

….....

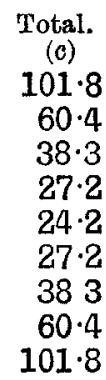

the intensity would be at different depths below "the surface, the gradual diminution being due to two causes-increase in distance from the anode and absorption of the rays by the superimposed tissues, both of which are taken into account in the data given. Column (a) represents the fall in intensity where the radiation is in one direction, and column (b) when the direction is reversed. The total in column (c) shows the variation throughout the tissues when the radiation is reversed in the manner indicated.

The question as to how nearly the $\mathrm{X}$ rays approach in penetrating power that of the hard gamma rays of radium cannot be answered without specifying the substance which is used to test the penetrating power. For example, if we make a comparison in this respect for lead we find that the $\mathrm{X}$ rays emitted from the Coolidge tube, under a spark-gap of $30 \mathrm{~cm}$. (points) and screened by nearly $2 \mathrm{~mm}$. of lead and $7 \mathrm{~mm}$. of aluminium, have about one-thirtieth of the penetrating power of hard gamma rays. If, however, the comparison be made with aluminium under the same conditions, the $X$ rays have a penetrating power of nearly one-fifth of that of the gamma rays, and nearly one-fourth if the comparison be made with water (tissues).

These observations do not preclude the possibility of still more penetrating $X$ rays being produced, but under the conditions specified the above figures represent what holds for the main part of the radiation; if harder $X$ rays are present they do not contribute more than about 1 part in 50 to the effects associated with the heavily screened radiation. By the term "hard gamma rays" is meant the radiation emitted from radium enclosed in a platinum tube $1 \mathrm{~mm}$. thick, the softer secondary rays from the platinum itself being absorbed by $2 \mathrm{~mm}$. of aluminium.

The investigations initiated by Lane in 1912 have been pursued in this country by W. H. Bragg and W. L. Bragg and by Moseley and Darwin, proving conclusively that the $X$ rays are ether vibrations, and many determinations of the wave-length of certain specified rays have now been made. The two latter investigators have shown that the wavelength of an $X$ ray may be inferred if the extent to which the ray is absorbed by some substance, such as aluminium, is known. There is a simple relation between the coefficient of absorption and the wavelength as long as one is not considering regions exhibiting selective absorption. We may make use of this relation to find the average wave-length of the different components constituting the heterogeneous beam whose absorption is represented by the curve (Fig. 1). Dividing this into sections in the way that analysis suggests that it should be done, it will be found that the wave-lengths for the three sections $D, E$, and $F$ are $4^{\circ} 85,3 \cdot 43$, and $2^{\cdot} 84 \times 10^{-9} \mathrm{~cm}$. respectively, and these are represented by the vertical lines on the lower diagram of Fig. 2, the length of the line

FIG. 2.

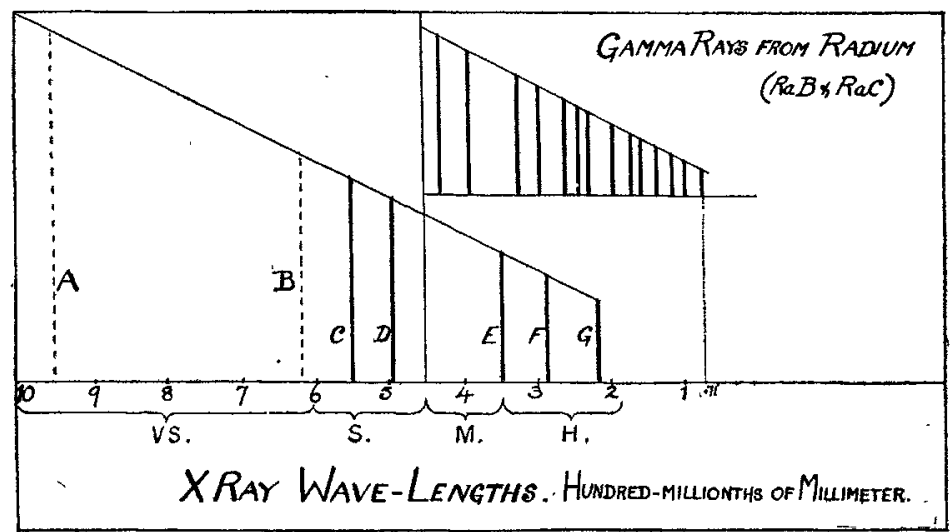

vs. $=$ very soft. s. $=$ soft. M. $=$ medium. H. $=$ hard.

being drawn proportional to the wave-length. Line $C$ is obtained as a result of measurements with a very soft bulb (about $6 \mathrm{~cm}$. spark-gap), and line $G$ with a wave-length of $2 \cdot 14 \times 10^{-9} \mathrm{~cm}$. represents the homogeneous very hard $X$ rays obtained under the conditions already specified. On the same diagram are inserted two wavelengths, A, B, which correspond to part of the characteristic radiation emitted by platinum and rhodium. The data for them are taken from "X Rays and Crystal Structure" (1915), by W. H. Bragg and W. L. Bragg. An inset in Fig. 2 shows the wave-lengths of the gamma rays emitted by radium ( $\mathrm{RaB}$ and $\mathrm{RaC}$ ) as determined by Rutherford and Andrade (vide Philosophical Magazine, August, 1914).

The representation in this manner-viz., $\mathrm{C}-\mathrm{G}-$ of the different varieties of $X$ rays must not be taken to mean that the rays emitted consist of homogeneous portions; the radiation is much more akin to white light, but certain regions appear homogeneous when tested by the method of absorption. The subdivisions "very soft," \&c., are quite arbitrary, and done for the purposes of the present article. It is hoped that the Dosage Committee of the Roentgen Society will make certain recommendations on this subject in the near future.

A comparison of the two sets of wave-lengths shows that the wave-length of the very hard $X$ rays (deduced from their absorption by aluminium) is about three times that of the shortest gamma ray measured by Rutherford and Andrade. With regard to the possibility of producing $X$ rays with the same wave-length as the hardest gamma rays, it would seem that the advent of the Coolidge tube should bring this realisation nearer than experience with the ordinary focus-tube suggested.

There is a tendency towards the more extensive use of hard screened $X$ rays. This brings with it the consideration that the operator is subject to an increasing amount of stray radiation. The yield of $X$ rays from modern bulbs is a very large one, and the question of protection of all those engaged in the administration of the rays becomes an increasingly difficult one. 